\title{
A Geometric Theory of Diblock Copolymer Phases
}

\author{
Gregory M. Grason, B. A. DiDonna, and Randall D. Kamien \\ Department of Physics and Astronomy, University of Pennsylvania, Philadelphia, PA 19104-6396, USA
}

(Dated: November 17, 2018)

\begin{abstract}
We analyze the energetics of sphere-like micellar phases in diblock copolymers in terms of wellstudied, geometric quantities for their lattices. We argue that the A15 lattice with $P m \overline{3} n$ symmetry should be favored as the blocks become more symmetric and corroborate this through a self-consistent field theory. Because phases with columnar or bicontinuous topologies intervene, the A15 phase, though metastable, is not an equilibrium phase of symmetric diblocks. We investigate the phase diagram of branched diblocks and find that the A15 phase is stable.
\end{abstract}

The ability to control the self-assembly of complex lattices by manipulating molecular architecture remains an essential aspect in the creation of new, functional materials. With only a few tunable parameters, diblock copolymer melts exhibit a wide variety of equilibrium phases which can be understood via the mean-field Gaussian chain model of "AB" diblock copolymers composed of immiscible A and B blocks [1, 2]. Indeed, in a system where the A and B-blocks are otherwise identical, there are only two thermodynamic variables, $\phi$, the volume fraction of $A$ type monomers, and $\chi N$, where $\chi$ is the Flory-Huggins parameter characterizing the repulsive interactions between the A- and B-type monomers and $N$ is the degree of polymerization [3]. In this letter, we present a model which predicts that the the A15 (shown in Fig. 19) lattice of diblocks is stable relative to other sphere-like phases for sufficiently large $\phi$ or, in other words, sufficiently symmetric diblocks. We corroborate this prediction by recalculating the phase diagram for symmetric diblocks (Fig. 10) via a self-consistent field theory (SCFT) for diblock copolymer melts [4].

The "classical" diblock phases are well-understood: near the order-disorder transition (ODT), Leibler developed a Landau-like theory in the weak-segregation regime to establish the stability of a body-centered cubic (BCC) phase, a hexagonal phase of columns and a lamellar phase 5]. Moreover, Semenov's picture of spherical micelles interacting through a disordered copolymer background when $\phi \ll 1$ accounts for the appearance of the facecentered cubic (FCC) lattice near the ODT in the meanfield phase diagram 6 ]. The more exotic gyroid phase was discovered [7, 8, 9] and was explained successfully by Matsen and Schick via SCFT [4]. In our study of the A15 lattice, we find that the hexagonal and gyroid phases intervene and thus there should be no stable A15 lattice for simple diblocks. However, sphere-like topologies are favored by branched diblock copolymers 10, 11, 12 and dendritic polymers 13, 14]; with this in mind we predict that sphere-like phases are stabilized and that the A15 phase is a ground state for this class of structures. By implementing, to our knowledge, the first full SCFT treatment of branched molecules (shown in Fig. 15) we have verified our theory.

In the dilute regime, Semenov's picture treats each mi- celle as an undistorted sphere so that the outer block extends to a spherical unit cell of radius $R_{S}$. This unit-cell approximation provides a lower bound for the free energy of the sphere-like phases [12]. However, when the micelles assemble into a periodic structure, the incompressibility of the melt allows no interstitial gaps and the spheres must deform into the Voronoi or Wigner-Seitz cells of the corresponding lattice. Our analysis will be in the strong-segregation limit, away from the ODT where fluctuation effects are less important. For small $\phi$, the A-block will form the center of each sphere-like micelle. As a gedanken experiment, we increase $\phi$ while maintaining the topology and keeping the A-block on the inside. In the most extreme limit, $\phi \rightarrow 1$ and the A-blocks are surrounded by a vanishingly thin B-block coat.

The free energy of strongly segregated diblock configurations of spherical geometry arises from the tension in the $\mathrm{AB}$ interface and the stretching of the polymers. Though it may appear that the curvature of the interface must be considered, in neat systems the curvature energy is completely accounted for by stretching through the incompressibility constraint [2]. The energy of the $\mathrm{AB}$ interface is proportional to its surface area $\Sigma$ (and $\sqrt{\chi}$ in the strong segregation limit [12, 15]). As illustrated in the insets to Fig. 2 when $\phi$ grows, the A-blocks in the center of the micelle fill a large volume and hence

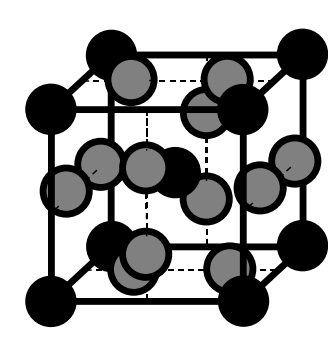

(a)

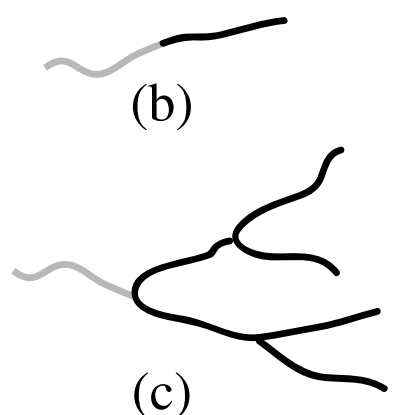

(c)
FIG. 1: In (a) we show the cubic unit cell of the A15 lattice. The black spheres form a BCC lattice and the A15 lattice has, in addition, dimers on each face, in grey. The diblock topologies we consider are (b) linear and (c) branched with three generations. 


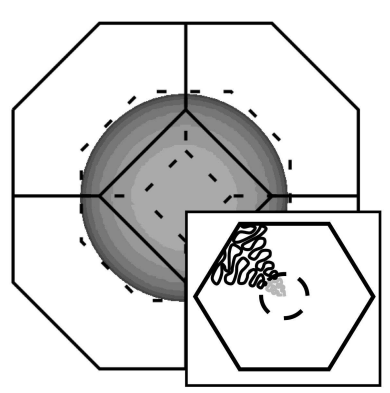

(a)

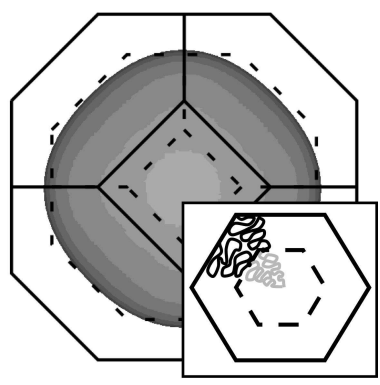

(b)
FIG. 2: A plot of the AB interfaces in the BCC $(\operatorname{Im} \overline{3} m)$ phase obtained by extracting the surface where the concentration of each block is equal. Both figures were extracted for $\chi N=40$. In (a) $\phi=0.222$ and the interface is almost spherical. In (b) $\phi=0.45$ and the interface takes on the shape of the Voronoi cell. The solids lines represent the entire BCC cell, while the dashed lines represent the reduced Voronoi cell that would arise in the $\phi \rightarrow 1$ limit. The insets depict (a) the spherical interface limit (small $\phi$ ) and (b) the flat interface limit (large $\phi)$.

the curvature of the $\mathrm{AB}$ interface is small. As a result, the interface is easily deformed and we expect that it will adopt the shape of the Voronoi cell. A dimensionless measure of the surface energy $\sqrt{\chi} \Sigma$ is

$$
\mathcal{A}=\frac{\Sigma}{\Sigma_{S}}
$$

where $\Sigma_{S}$ is the area of the interface for a spherical micelle of the same volume. This ratio may be rewritten as $\mathcal{A}_{X}=\gamma(X) /(36 \pi)^{1 / 3}$ where $\gamma(X)$ depends on the lattice $X$ and relates the area to the volume $V$ of the Voronoi cell: $\Sigma=\gamma(X) V^{2 / 3}$. It is conjectured that an A15 lattice of equal volume cells minimizes $\gamma[16$ and it is known that $\gamma(\mathrm{A} 15)=5.297<5.315=\gamma(\mathrm{BCC})<\gamma(\mathrm{FCC})=5.345$ [17, 18]. This fact can be used to argue the stability of the A15 phase in fuzzy colloidal systems [19] and is crucial to the analysis here. The area per unit volume scales as $R_{S}^{-1}$ and so the surface tension contributes $\mathcal{A} / R_{S}$ to the free energy density.

The second contribution to the free energy arises from the stretching of the polymers to fill the cell. Due to incompressibility, the number of chains in each wedge of solid angle $d \Omega$ is proportional its volume, $\frac{1}{3} R^{3}(\Omega) d \Omega$, where $R(\Omega)$ is the distance to the boundary. Since the stretching energy for both blocks of chains in this wedge is proportional to $R^{2}(\Omega)$ [15],

$$
\mathcal{I}_{X}=\frac{\int_{\Pi} d \Omega R(\Omega)^{5}}{4 \pi R_{S}^{5}}
$$

is a dimensionless measure of the stretching relative to the stretching in a sphere of radius $R_{S}$ with the same volume as the Voronoi cell $\Pi$. This integral is propor- tional to the moment:

$$
G(X)=\frac{\int_{\Pi} d^{3} R R^{2}}{3\left(\int_{\Pi} d^{3} R\right)^{5 / 3}}=\frac{3^{2 / 3}}{5(4 \pi)^{2 / 3}} \mathcal{I}_{X}
$$

which has been well-studied 20] in the context of the quantizing problem: $G(\mathrm{BCC})=0.078543<0.078745=$ $G(\mathrm{FCC})=G(\mathrm{~A} 15)$ 21]. The stretching energy per chain (or per unit volume) scales as $R_{S}^{2}$ and so the free energy contribution from stretching is proportional to $\mathcal{I} R_{S}^{2}$. The prefactor of this term will, in general, depend on the topology of the micelles and $\phi$ [15] but for the structures we consider, this prefactor does not change.

Putting these two effects together, we find a Flory-like free energy density:

$$
f \sim \frac{\mathcal{A}_{X}}{R_{S}}+\mathcal{I}_{X} R_{S}^{2}
$$

Minimizing over $R_{S}$, we find that $f=f_{0}\left[\mathcal{I}_{X} \mathcal{A}_{X}^{2}\right]^{1 / 3} \propto$ $\gamma^{2 / 3} G^{1 / 3}$ where $f_{0}$ is the energy of the spherical micelle 15]. It follows, for instance, that as $\phi$ grows, the $\mathrm{BCC}$ lattice is lower in energy than the FCC lattice since $\gamma(\mathrm{BCC})<\gamma($ FCC $)$ 12]. We find that the A15 lattice is lower in energy still: $f_{A 15}=1.0707 f_{0}<1.0722 f_{0}=$ $f_{B C C}<f_{F C C}=1.0772 f_{0}$. It should be noted that a more detailed, self-consistent treatment 12] yields the same free energy (4) and the same numerical results given here for the BCC and FCC lattices. We have thus established a fundamental and unavoidable geometric frustration between the stretching and interfacial energies - the BCC lattice minimizes $G$ and the A15 lattice minimizes $\gamma$. We note that since the A15 lattice has two distinct sites, it is possible for their corresponding cells to adopt different volumes to lower the energy further. Indeed, by adjusting the Voronoi cells $G(\mathrm{~A} 15)$ and $\gamma(\mathrm{A} 15)$ can be lowered [17, 21], ultimately lowering $f_{A 15}$ by a further $0.03 \%$.

As $\phi$ decreases the minority region shrinks and the curvature grows, leading to a larger restoring force to the optimal, spherical shape. In this case the connectivity of the A-blocks to the B-blocks would require a more detailed analysis, such as the "kinked path" ansatz of Milner and Olmsted 12. Since the BCC lattice minimizes the stretching energy it is not surprising that it is the equilibrium phase at smaller $\phi$.

Because we assumed that the $\mathrm{AB}$ interface is perfectly flat, our calculation puts upper limits on the free energies of these sphere-like phases. Nonetheless, our argument suggests that as $\phi$ grows, the A15 phase should become the more stable sphere-like phase. To critically test our theory, we employed the SCFT implementation of Matsen and Schick to compare the free energy of a phase with the $P m \overline{3} n$ symmetry to the free energy of the other sphere-like phases of diblock melts [4]. This scheme has the advantage of providing numerically exact results using a Fourier basis of a given symmetry 


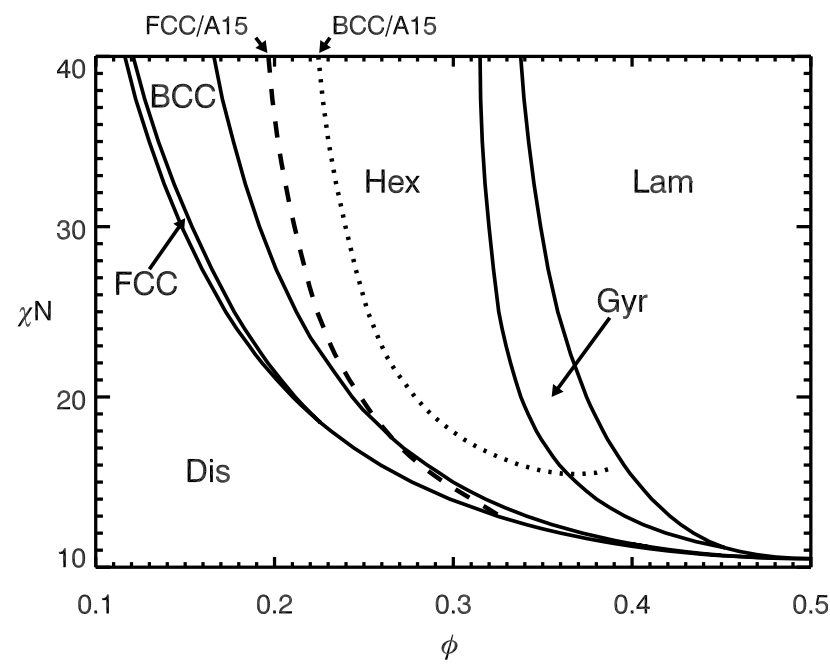

(a)

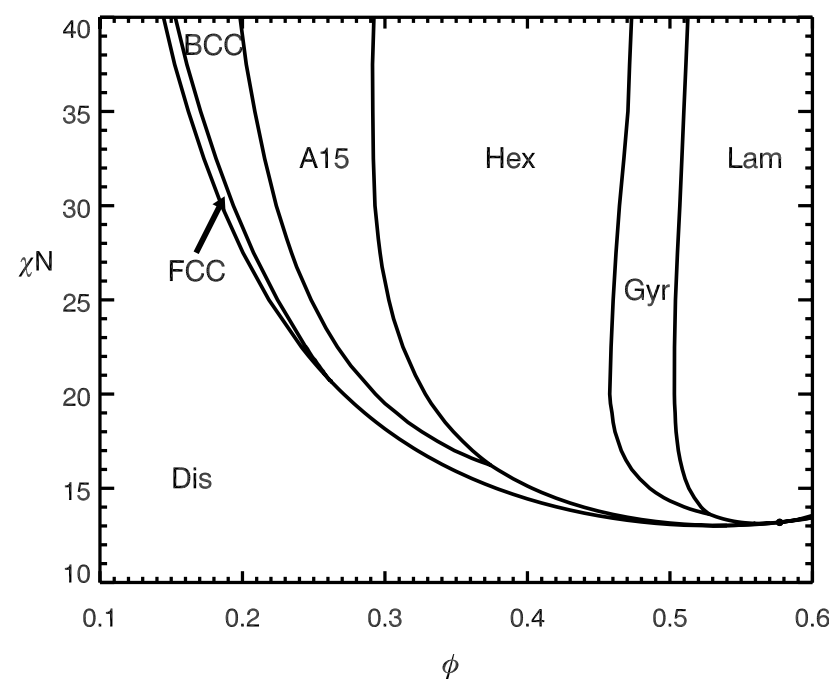

(b)

FIG. 3: The SCFT phase diagram for melts composed of (a) linear chains. The dotted line is where the free energy of the BCC phase is equal to that of the $\mathrm{A} 15$ phase, while the dashed line is the corresponding boundary for the FCC phase. In the phase diagram (b) for the three-generation diblocks shown in Fig. 11 the A15 phase is a ground state for a significant range of $\phi$ and $\chi N$. All the B blocks are of equal length. Note the critical point on the lamellar/disordered boundary.

and requires significantly less computing power than is required to achieve an equivalent precision with a numerical real-space approach 22]. The efficiency of this method allows us to explore the entire phase diagram and, because we choose the symmetry of the basis we can quickly compare three-dimensional lattices of different symmetries, even in regimes where columnar, lamellar and gyroid phases are the ground states [23]. Note that when considering close-packed lattices, hexagonal close-packed and FCC lattices have degenerate free energies and so we use the $F m \overline{3} m$ basis only. The first result of our calculation shows that as $\phi \rightarrow 0$ the $\mathrm{AB}$ interface is spherical and as $\phi$ grows, the interface deforms significantly into the shape of the Voronoi cell, as shown in Fig. 2 This confirms our intuitive discussion about the AB interface.

In order to compute the free energy of all phases within $0.005 \%$ for $\chi N \leq 40$ we required up to 760 basis functions. This level of precision allowed us to delineate the phase boundaries to within \pm 0.001 for $\phi$ and \pm 0.01 for $\chi N$. Our calculation corroborates our Flory-like theory and shows that the A15 lattice is the most stable threedimensional lattice for sufficiently large $\phi$, as shown in Fig. 37. In addition to the known phases for symmetric diblock copolymers, we also computed the boundaries at which the free energies of the three candidate sphere-like phases crossover. We have reproduced the boundary between the BCC and FCC phases reported by Matsen and Bates [23], and have also found the boundaries at which the free energy of the A15 lattice becomes lower than that of the of FCC and BCC phases, respectively. Thus, we see that the A15 lattice is the stable sphere-like phase as $\phi \rightarrow 1$. Note that the boundary between BCC and A15 terminates at $\phi=0.390$ and $\chi N=15.86$ because at this level of segregation for $\phi>0.390$ the $\operatorname{Im} \overline{3} m$ sphere-like phase melts.

Having considered the A15 lattice, it is natural to consider other periodic arrangements of spheres. We used the same SCFT method to compute the free energy of sphere-like micelles assembled into the simple cubic (SC) and diamond (D) lattice, with $P m \overline{3} m$ and $F d \overline{3} m$ symmetries respectively. For example, at $\chi N=17.0$ and $\phi=0.26$ the free energies per chain in the BCC, FCC, A15, SC and D phases are 3.2455, 3.2459, 3.2461, 3.2568 and 3.2701 , respectively in units of $k_{B} T$. This trend continues into the region where sphere-like phases are not stable: at $\chi N=17.0$ and $\phi=0.31$ the free energies per chain of the A15, BCC, and FCC phases are 3.6107, 3.6120 , and 3.6158, respectively. At this point of the phase diagram the free energy per chain of the SC phase is 3.6295 and the $\mathrm{D}$ phase of spheres becomes unstable. The free energies of the BCC, FCC, and A15 phases remain within $1 \%$ of each other over the entire region of the phase space we explored while the SC and D sphere-like phases are never the lowest energy phases.

Though the A15 lattice will not be observed in neat linear diblock melts, our calculation suggests that if the sphere-like phases were stabilized it would persist as an equilibrium state. One way to stabilize sphere phases for more symmetric diblocks is to change the architecture of the molecule. For instance, if one block of the copolymers is branched, the stretching energy combined with incompressibility will favor dividing surfaces which are curved over a substantial portion of the phase dia- 
gram. This was predicted by Olmsted and Milner for miktoarm block copolymers [12] and later observed [10]. Similarly, calculations by Pickett for multiply branched diblocks predict the same generic effect 11]. We have implemented a SCFT algorithm to explore the phase diagram of branched diblocks with a linear A-block connected to $n$ B-blocks, each of which are connected to $n$ B-blocks, etc. for $g$ generations so that the final generation has $(g-1) n$ B-blocks (the $g=1$ system is pure A). We will report elsewhere on the details of our calculation [24] but it closely follows the original analysis of Matsen and Schick [4]. Because of the time required and numerical intensity of the calculation, we have focussed first on a $g=3$ generation diblock with $n=2$ blocks at each branch as a "proof-of-principle" for both our theoretical argument and our algorithm. As shown in Fig. [3p, we find that sphere-like phases are stable over a greater range of $\phi$ and that the $\mathrm{A} 15$ is a stable phase at values of $\phi$ larger than those for which the BCC phase is stable - precisely what we would expect from our geometric theory. It is also worth noting that for this region of the phase diagram, the gyroid is the equilibrium structure for this system, and the double-diamond or hexagonallyperforated lamellar phases are only metastable as in linear diblocks.

By employing purely geometric quantities we have argued that the A15 lattice should be the lowest energy sphere-like micelle phase of diblock copolymers as the blocks become more and more symmetric. Through a SCFT calculation we have corroborated our theory though we found that where the A15 lattice was stable, non-spherical columnar or gyroid phases were the ground states of the diblock melt. By considering branched diblocks which favor sphere-like phases, we have found via SCFT that the A15 is an equilibrium phase of branched diblocks with sufficiently large (three) generations. Further work will fully map out the phase diagram of branched diblocks as function of molecular geometry and topology.

It is a pleasure to thank V. Percec, A. Yodh and P. Ziherl for stimulating discussions. We are indebted to D. Duque, M. Matsen and M. Schick for supplying us with their numerical code. This work was supported by NSF Grant DMR01-02459, the Donors of the Petroleum Research Fund, Administered by the American Chemical Society and a gift from L.J. Bernstein.
[1] F. S. Bates, Science, 251, 898 (1991).

[2] M. W. Matsen, J. Phys.: Cond. Matt., 14, R21 (2002).

[3] F. S. Bates and G. H. Fredrickson, Ann. Rev. Phys. Chem., 41, 525 (1990).

[4] M. W. Matsen and M. Schick, Phys. Rev. Lett., 72, 2660 (1994).

[5] L. Leibler, Macromolecules, 13, 1602 (1980).

[6] A. Semenov, Macromolecules, 22, 2849 (1989.

[7] D. A. Hajduk, P. E. Harper, S. M. Gruner, C. C. Honeker, E. L. Thomas, and L. J. Fetters, Macromolecules, 27, 4063 (1994).

[8] D. A. Hajduk, H. Takenouchi, M. A. Hillmyer, F. S. Bates, M. E. VIgild, and K. Almdal, Macromolecules, 30, 3788 (1997).

[9] D. A. Hajduk, P. E. Harper, S. M. Gruner, C. C. Honeker, E. L. Thomas, and L. J. Fetters, Macromolecules, 26, 956 (1995).

[10] F. L. Beyer, S. P. Gido, G. Velis, N. Hadjichristidis, and N. B. Tan, Macromolecules, 32, 6604 (1999).

[11] G. T. Pickett, Macromolecules, 35, 1896 (2002).

[12] P. D. Olmsted and S. T. Milner, Macromolecules, 31, 4011 (1998).

[13] V. S. K. Balagurusamy, G. Ungar, V. Percec, and G. Johansson, J. Am. Chem. Soc., 119, 1539 (1997).

[14] V. Percec, C.-H. Ahn, G. Ungar, D. J. P. Yeardly, M. Möller, and S. S. Sheiko, Nature, 391, 161 (1998).

[15] P. D. Olmsted and S. T. Milner, Phys. Rev. Lett., 72, 936 (1994).

[16] D. Weaire and R. Phelan, Philos. Mag. Lett., 69, 107 (1994).

[17] R. Kusner and J.M. Sullivan, Forma, 11, 233 (1996).

[18] Since the A15 lattice has two distinct Voronoi cells, the lattice quantities $\gamma(A 15)$ and $G(A 15)$ represent the weighted average of the two cell types.

[19] P. Ziherl and R. D. Kamien, Phys. Rev. Lett., 85, 3528 (2000).

[20] J. H. Conway and N. J. A. Sloane, Sphere Packings, Lattices and Groups, Third Ed., (Springer-Verlag, New York, 1999).

[21] N. Kashyap and D. L. Neuhoff, IEEE Trans. Inform. Theory, 47, 2538 (2001).

[22] F. Drolet and G. H. Fredrickson, Phys. Rev. Lett., 83, 4317 (1999).

[23] M. W. Matsen and F. S. Bates, Macromolecules, 29, 1091 (1996).

[24] G. M. Grason, B. A. DiDonna, R. D. Kamien, unpublished. 